\title{
INDIAN OCEAN MAPOURIA SPECIES TRANSFERRED TO PSYCHOTRIA (RUBIACEAE-PSYCHOTRIEAE)
}

\author{
AARON P. DAVIS, RAFAËL GOVAERTS \& MARIE BRIGGS
}

The Herbarium, Royal Botanic Gardens, Kew, Richmond, Surrey, TW9 3AE, United Kingdom

\section{SUMMARY}

Mapouria Aubl. is a synonym of Psychotria L. In Madagascar and the Comoros there are 67 species of Mapouria that fall within the circumscription of Psychotria and correspondingly 64 species (67 taxa) require transfer to Psychotria. Thirty-seven new combinations and 30 new names are proposed; five lectotypes are designated.

Key words: Psychotrieae, Mapouria, Psychotria, Comoros, Madagascar.

\section{INTRODUCTION}

Mapouria Aubl. was described by Aublet (1775a), based on M. guianensis Aubl. collected from French Guiana. Of the features listed by Aublet, deciduous stipules, terminal paniculate inflorescences, heterostylous flowers, and a 5-lobed calyx are amongst those diagnostic for the genus. The plate is figured in vol. 3 of Aublet's 'Histoire des Plantes de la Guiane Françoise '(1775b: pl. 67).

In his summary on the identity of the genera Mapouria and Grumilea Gaertn., Bremekamp (1961:319) stated that Mapouria should be applied to members of the tribe Psychotrieae that possess deciduous stipules, heterostylous flowers, pyrenes lacking a longitudinal intrusion on the commissural (ventral/adaxial) surface, and endosperm that is either ruminate on the adaxial surface only or extends over the whole surface. Bremekamp asserted that Mapouria, which was formerly used only for New World Psychotrieae, should be used for species that were previously included in Grumilea, and that Mapouria need not be given up in favour of Psychotria. On this basis, Bremekamp (1963) enumerated 67 species of Mapouria from Madagascar and the Comoros, mostly described as new species but some were transferred from Psychotria.

Following Bremekamp (1963), Petit (1964) wrote his revision of continental African Psychotria. In this work Petit clearly and succinctly stated that there are few, if any, differences between Mapouria and Psychotria, and specifically that the characterization for Mapouria as given by Bremekamp (1963), and as outlined above, included many of the African species. Petit (1964: 24) averred that Mapouria should not be upheld. This view was supported by Steyermark (1972: 444-446). Some years later, Hamilton (1989) formally placed Mapouria into the synonymy of Psychotria, a decision that has been widely accepted (Taylor, 1996; Andersson, 2002). Andersson (2002) sampled 
two species of Madagascan Mapouria (M. ankafinensis K. Schum. and M. macrochlamys Bremek.) in his assessment of the Psychotria complex (Psychotrieae), using data from the rps 16 intron. Mapouria macrochlamys (Mapouria series VI) was placed in Psychotria s.s. clade III, with the neotropical type of Mapouria, and M. ankafinensis (Mapouria series III) was unresolved in Psychotria s.s. Despite the paucity of molecular information for Indian Ocean Mapouria, the data provided by Andersson (2002) is consistent with the placement of Mapouria as a synonym of Psychotria.

Nearly all species of Old World Mapouria have now been placed in Psychotria or other genera of Psychotrieae (see www.rbgkew.org.uk/wcsp/rubiaceae). Conspicuously, there remain 67 Indian Ocean species of Mapouria. After 1) careful review of the generic and species descriptions (Bremekamp, 1963); 2) examination of type specimens and/or other authentic material held at $\mathrm{K}$ and $\mathrm{P}$ (abbreviations after Holmgren et al., 1990); and 3) field study (by A. Davis), it is clear that these Mapouria species conform to characterization of Psychotria (Petit, 1964; Davis et al., 2001; Andersson, 2002). We therefore take the opportunity to transfer 67 species of Indian Ocean Mapouria to Psychotria. We have maintained the species delimitations made by Bremekamp (1963) for the 67 Mapouria species and believe that at the present time there is no need for species-level synonymy. Despite identifying numerous new collections of Madagascan Mapouria in the last ten years we have so far identified no potential synonyms. Indeed, it is likely that there are several new species that require formal description. Indian Ocean Mapouria mostly have terminal inflorescences and yellow corollas, a combination of characters that is also present in some species of Africa Psychotria, such as $P$. capensis (Eckl.) Vatke. However, there is also a group of species with axillary or near-axillary inflorescences, that have white, red or reddish corollas (Mapouria series I of Bremekamp, 1963). Axillary inflorescences are rare in Psychotria and the Psychotrieae (A. Davis, unpubl. data) but otherwise these species conform to African Psychotria (Petit, 1964).

In addition to the taxonomic treatment of Mapouria and Psychotria by Bremekamp (1963), he also revised four other Psychotrieae genera occurring in Madagascar and the Comoros, viz. Apomuria Bremek., Trigonopyren Bremek., Carinta W. Wight and Psathura Comm. ex A. Juss. Carinta is a synonym of Geophila D. Don, but the others are accepted names. Apomuria and Trigonopyren seem to fall within the delimitation of Psychotria, resembling some of the species from continental Africa in general morphology and pyrene structure. Psathura has a multilocular ovary, with 3-6 locules, but otherwise has the potential to be incorporated within Psychotria. Further work is required to ascertain the taxonomic placement of these three genera.

\section{METHODS}

Herbarium material was consulted at the Muséum National d'Histoire Naturelle, Paris (P), and the Royal Botanic Gardens, Kew (K). All specimens cited here have been seen by us, unless indicated otherwise (non vidi: n.v.). Some species were examined in the field in Madagascar, during eight field trips over a nine year period (1996-2004) by A. Davis (unpubl. data). Pyrenes were prepared by immersing fruits in boiling water for three to five minutes, depending on the size and thickness of the fruit. The mesocarp 
(and any other soft tissue) was removed using a blunt scalpel blade and blunt needle. The presence vs absence of preformed germination slits (PGSs) was assessed upon removal of the mesocarp. The endosperm was examined by cutting the pyrene in transverse section with a sharp scalpel. To test for an ethanol soluble pigment, a single seed, or one half of a seed, was placed into a test-tube and covered with $70 \%$ ethanol for c. 5 minutes, at room temperature. The resulting solution was held against a white piece of paper and the colour assessed. On completion of the test, the seeds were dried and returned to their respective herbarium sheets. Pyrene observations were made using a Leica MZ95 stereomicroscope.

\section{RESULTS}

\section{PYRENE MORPHOLOGY}

All species formerly included in Mapouria from Madagascar and the Comoros have the same basic pyrene structure, which is the same as most Africa Psychotria: the pyrenes lack preformed germination slits (PGSs; see Petit, 1964; Robbrecht, 1988, 1989; Davis et al., 2001; Piesschaert, 2001), the endosperm is ruminate and the testa has an ethanol (70\%) soluble pigment (see Petit, 1964), which turns ethanol red (see Methods). According to Bremekamp $(1963: 6,8)$ the lack of a longitudinal intrusion on the inner (adaxial) face of each pyrene (as present in some Psychotria spp.) is a key feature of Mapouria. Indeed, the pyrenes of most species of Madagascan Mapouria examined by us are like this, but there are also many species of continental African Psychotria (Petit, 1964) and Asian Psychotria (A. Davis, pers. observ.) that lack a longitudinal intrusion on the inner face of each pyrene.

\section{TRANSFER OF INDIAN OCEAN MAPOURIA TO PSYCHOTRIA}

For the transfer of Indian Ocean Mapouria species given below we have retained the species in the same number order as Bremekamp (1963), and have kept the species within their respective informal series groups (Series I-VII). This will make it easier to use the identification keys provided by Bremekamp (1963), which still have considerable utility.

\section{SERIES I}

1. Psychotria bremekampiana A.P. Davis \& Govaerts, nom. nov.

Mapouria discolor Bremek. (1963) 23, non Psychotria discolor (Griseb.) Rolfe (1893) 258. - Type: Humbert, Capuron \& Cours 24786 (holo P), Madagascar (Nord-est), massif de l'Anjanaharibe, à l'ouest d'Andapa (haute Andramonta, bassin de la Lokoho), $1800 \mathrm{~m}$.

Distribution - NE Madagascar.

Note - Named after the Dutch taxonomist and Rubiaceae specialist C.E.B. Bremekamp. 
2. Psychotria andapae A.P. Davis \& Govaerts, nom. nov.

Mapouria lokohensis Bremek. (1963) 24, non Psychotria lokohensis Bremek. (1960) 50. - Type: Humbert \& Capuron 22039 (holo P), Madagascar, limite des Domaines de l'Est et du Centre, environs d'Andapa, bassin de la Lokoho, collines de l'Ankobahina, affluent de l'Ankasahana.

Distribution - NE Madagascar.

3. Psychotria antsirananensis A.P. Davis \& Govaerts, nom. nov.

Mapouria montana Bremek. (1963) 25, non Psychotria montana Blume (1823) 54. - Type: Perrier de la Bâthie 16429 (holo P), Madagascar, Domaine du Centre, massif du Tsaratanana, vers $2000 \mathrm{~m}$.

Distribution - N Madagascar.

4. Psychotria rosella (Bremek.) A.P. Davis \& Govaerts, comb. nov.

Basionym: Mapouria rosella Bremek. (1963) 25. - Type: Humbert, Capuron \& Cours 24498 (holo P), Madagascar (Nord-est), massif de l'Anjanaharibe, à l'ouest d'Andapa (bassin de la Lokoho), $700 \mathrm{~m}$.

Distribution - NE Madagascar.

5. Psychotria capuronii A.P. Davis \& Govaerts, nom. nov.

Mapouria suffruticosa Bremek. (1963) 26, non Psychotria suffruticosa Müll.Arg. (1881) 249. - Type: Humbert \& Capuron 22120 (holo P), Madagascar (Nord-est), pentes occidentales du massif de Marojejy, bassin de la Lokoho, à l'est d'Ambalamanasy II, district d'Andapa, 450-800 m.

Distribution - NE Madagascar.

Note - Named after French botanist R. Capuron in recognition of his considerable contribution to the botany of Madagascar.

6. Psychotria brachyblastus A.P. Davis \& Govaerts, nom. nov.

Mapouria perrieri Bremek. (1963) 27, non Psychotria perrieri Bremek. (1963) 150. - Type: Perrier de la Bâthie 15381 (holo P; iso K, P), Madagascar, Domaine du Centre, massif du Tsaratanana, $2000 \mathrm{~m}$.

Distribution - N Madagascar.

7. Psychotria oreotrephes (Bremek.) A.P. Davis \& Govaerts, comb. nov.

Basionym: Mapouria oreotrephes Bremek. (1963) 28. - Type: Perrier de la Bâthie 3748 (holo P), Madagascar, Domaine du Centre, massif du Tsaratanana, $2000 \mathrm{~m}$.

Distribution - N Madagascar.

8. Psychotria glaucifolia A.P. Davis \& Govaerts, nom. nov.

Mapouria glaucescens Bremek. (1963) 29, non Psychotria glaucescens Kunth (1819) 358. - Type: Perrier de la Bâthie 3731 (holo P), Madagascar, Domaine du Centre, massif du Tsaratanana, $2500 \mathrm{~m}$.

Distribution - N Madagascar. 
9. Psychotria maningoryensis A.P. Davis \& Govaerts, nom. nov.

Mapouria himanthophylla Bremek. (1963) 30, as 'himantophylla', non Psychotria himanthophylla Bremek. (1960) 53. - Type: Homolle 521 (holo P; iso P), Madagascar, limite des Domaines de l'Est et du Centre, Lac Alaotra, entre Menasaka et Ambodiriana, aux bords du Maningory.

Distribution - Central Madagascar.

10. Psychotria paradoxalis (Bremek.) A.P. Davis \& Govaerts, comb. nov.

Basionym: Mapouria paradoxalis Bremek. (1963) 31. - Type: Humbert 23081 (holo P), Madagascar, limite des Domaines de l'Est et du Centre, contreforts occidentaux du massif de Marojejy, près du col de Doanyanala (limite des bassins de la Lokoho et de l'Andraronga).

Distribution - NE Madagascar.

11. Psychotria maroensis (Bremek.) A.P. Davis \& Govaerts, comb. nov.

Basionym: Mapouria maroensis Bremek. (1963) 32. - Type: Mocquerys 342 (holo G n.v.; iso P n.v.), Madagascar, Domaine de l'Est, Baie d'Antongil, Maroa.

Distribution - NE Madagascar.

\section{SERIES II}

12. Psychotria pyrrotricha (Bremek.) A.P. Davis \& Govaerts, comb. nov.

Basionym: Mapouria pyrrotricha Bremek. (1963) 32. - Type: Perrier de la Bâthie 12960 (holo P), Madagascar, Domaine du Centre, Est-Imerina, prov. Tananarive, près de Manjakandriana, Mont Lohavohitra, $1500 \mathrm{~m}$.

Distribution - Central Madagascar.

13. Psychotria rakotonasoloi A.P. Davis \& Govaerts, nom. nov.

Mapouria longipedunculata Bremek. (1963) 34, non Psychotria longipedunculata (Gardner) Müll. Arg. (1881) 237. - Type: Cours 2367 (holo P), Madagascar, Limites des Domaines du Centre et de l'Est, entre Manakambahiny et Nonokambo, $1200 \mathrm{~m}$.

Distribution - Central and E Madagascar.

Note - Named after Malagasy botanist and Rubiaceae specialist F. Rakotonasolo.

14. Psychotria andriantiana A.P. Davis \& Govaerts, nom. nov.

Mapouria marojejensis Bremek. (1963) 35, non Psychotria marojejensis Bremek. (1963) 144. - Type: Humbert 23726 (holo P), Madagascar (Nord-est), pente orientale du massif de Marojejy, à l'ouest de la rivière Manantenina, affluent de la Lokoho, 1850-2150 m.

Distribution - NE and Central Madagascar.

Note - Named after Malagasy botanist J. Andriantiana. 
15. Psychotria ankafinensis (K. Schum.) A.P. Davis \& Govaerts, comb. nov.

Basionym: Uragoga ankafinensis K. Schum. (1899) 106. - Mapouria ankafinensis (K. Schum.) Bremek. (1963) 36. - Type: Hildebrandt 3939 (holo P; iso K, P), Madagascar, Domaine du Centre, Sud Betsileo, forêt d'Ankafina.

Distribution - Central Madagascar.

16. Psychotria baronii (Bremek.) A.P. Davis \& Govaerts, comb. nov.

Basionym: Mapouria baronii Bremek. (1963) 37, as ‘baroni’. - Type: Baron 157 (holo K), Madagascar, Domaine du Centre, Betsileo.

Distribution - Central Madagascar.

17. Psychotria ambongensis (Bremek.) A.P. Davis \& Govaerts, comb. nov.

Basionym: Mapouria ambongensis Bremek. (1963) 38. - Type: Boivin s.n. (holo P), Madagascar, Domaine de l'Ouest, Ambongo.

Distribution - NW Madagascar.

\section{SERIES III}

18. Psychotria cephaloides A.P. Davis \& Govaerts, nom. nov.

Mapouria capitata Bremek. (1963) 39, non Psychotria capitata Ruiz \& Pav. (1799) 59. - Type: Humblot 556 (holo P; iso K, P), Madagascar, without further indication of locality.

Distribution - Madagascar.

19. Psychotria humblotii (Bremek.) A.P. Davis \& Govaerts, comb. nov.

Basionym: Mapouria humblotii Bremek. (1963) 40. - Type: Humblot 85 (holo P), Madagascar, without further indication of locality.

Distribution - Madagascar.

20. Psychotria nossibensis A.P. Davis \& Govaerts, nom. nov.

Mapouria richardiana Bremek. (1963) 41, non Psychotria richardiana Urb. (1913) 472. - Type: Boivin 2053 (holo P; iso P), Madagascar, Domaine du Sambirano, Nossi-Bé, forêt de Loucoube [Lokobe].

Distribution - N Madagascar.

20a. var. nossibensis

Distribution - N Madagascar.

20b. var. arrhyncha (Bremek.) A.P. Davis \& Govaerts, comb. nov.

Basionym: Mapouria richardiana Bremek. var. arrhyncha Bremek. (1963) 41. - Type: Boivin 2054 (holo P; iso P), Madagascar, Domaine du Sambirano, Nossi Bé, forêt de Lokoube [Lokobe].

Distribution - N Madagascar. 
21. Psychotria megalocarpa (Bremek.) A.P. Davis \& Govaerts, comb. nov.

Basionym: Mapouria megalocarpa Bremek. (1963) 41. - Type: Viguier \& Humbert 517 (holo P), Madagascar, Domaine de l'Est, prov. d'Andovoranto, distr. d'Anivorano, pic de Vohilonjo, près de Féhomby, $400 \mathrm{~m}$.

Distribution - E Madagascar.

\section{SERIES IV}

22. Psychotria mangorensis (Bremek.) A.P. Davis \& Govaerts, comb. nov.

Basionym: Mapouria mangorensis Bremek. (1963) 43. - Type: Perrier de la Bâthie 18059(holo P), Madagascar, Domaine de l'Est, bassin inférieur du Mangoro.

Distribution - E Madagascar.

23. Psychotria betamponensis (Bremek.) A.P. Davis \& Govaerts, comb. nov.

Basionym: Mapouria betamponensis Bremek. (1963) 43. - Type: Decary 16925 (holo P), Madagascar, Domaine de l'Est, Betampona, Rés. Nat. I.

Distribution - E Madagascar.

24. Psychotria onivensis (Bremek.) A.P. Davis \& Govaerts, comb. nov.

Basionym: Mapouria onivensis Bremek. (1963) 44. - Type: Perrier de la Bâthie 17054 (holo P), Madagascar, limite des Domaines de l'Est et du Centre, forêt d'Andasibe sur l'Onive, vers $800 \mathrm{~m}$.

Distribution - E and Central Madagascar.

25. Psychotria menalohensis (Bremek.) A.P. Davis \& Govaerts, comb. nov.

Basionym: Mapouria menalohensis Bremek. (1963) 45. - Type: Cours 1980 (holo P; iso P), Madagascar, Domaine de l'Est, petite forêt de la Menaloha, au bord de la route de Nickelville, $850 \mathrm{~m}$.

Distribution - E Madagascar.

\section{SERIES V}

26. Psychotria anderssoniana A.P. Davis \& Govaerts, nom. nov.

Mapouria dolichocarpa Bremek. (1963) 46, non Psychotria dolichocarpa K. Schum. (1898) 686. - Type: Boivin s.n. (holo P), Madagascar, Domaine du Sambirano, Nossi-bé, forêt de Loucoube [Lokobe].

Distribution - N Madagascar.

Note - This species is named in memory of the Swedish botanist and Rubiaceae specialist Professor Lennart Andersson (1948-2005).

27. Psychotria anjouanensis A.P. Davis \& Govaerts, nom. nov.

Mapouria lavanchiei Bremek. (1963) 47, non Psychotria lavanchiei Bremek. (1963) 143. - Type: Lavanchie 17 (holo P), Comores, Anjouan.

Distribution - Comoros (Anjouan). 
28. Psychotria rubropedicellata (Bremek.) A.P. Davis \& Govaerts, comb. nov.

Basionym: Mapouria rubropedicellata Bremek. (1963) 47. - Type: Perrier de la Bâthie 3788 (holo P), Madagascar, Domaine de l'Ouest, Ambongo, Namoroka, près d'Andranomavo.

Distribution - W Madagascar.

29. Psychotria sonocorova (Bremek.) A.P. Davis \& Govaerts, comb. nov.

Basionym: Mapouria sonocorova Bremek. (1963) 48. - Type: Humbert \& Capuron 28950 (holo P), Madagascar, Domaine de l'Est (Sud), Fort Dauphin, Bevilany à l'ouest de Fort Dauphin, $100-200 \mathrm{~m}$.

Distribution - SE Madagascar.

30. Psychotria aegialodes (Bremek.) A.P. Davis \& Govaerts, comb. nov.

Basionym: Mapouria aegialodes Bremek. (1963) 49. - Type: Decary 10318 (holo P; iso P), Madagascar, Domaine de l'Est (Sud), Fort Dauphin, Vinanibe, forêt littorale.

Distribution - SE Madagascar.

\section{SERIES VI}

31. Psychotria rufovillosa (Bremek.) A.P. Davis \& Govaerts, comb. nov.

Basionym: Mapouria rufovillosa Bremek. (1963) 50. - Type: Humblot 480 (holo P; iso K, P), Madagascar, Domaine de l'Est, Antsianaka.

Distribution - E Madagascar.

32. Psychotria macrochlamys (Bremek.) A.P. Davis \& Govaerts, comb. nov.

Basionym: Mapouria macrochlamys Bremek. (1963) 51. - Type: Decary 7116 (holo P), Madagascar (Est), sud de Moramanga.

Distribution - E Madagascar.

33. Psychotria tolongoinensis A.P. Davis \& Govaerts, nom. nov.

Mapouria erythrotricha Bremek. (1963) 52, non Psychotria erythrotricha Elmer (1913) 1891. - Type: SF 7131 (holo P), Madagascar, Domaine de l'Est, Fort Carnot - Tolongoina - Amelatsily.

Distribution - E Madagascar.

34. Psychotria integristipulata A.P. Davis \& Govaerts, nom. nov.

Mapouria subrufa Bremek. (1963) 53, non Psychotria subrufa Miq. (1857) 292. - Type: Perrier de la Bâthie 15238 (holo P; iso P), Madagascar, Domaine du Centre, Mt Tsaratanana, $1200 \mathrm{~m}$.

Distribution - N Madagascar. 
35. Psychotria manongarivensis A.P. Davis \& Govaerts, nom. nov.

Mapouria puberula Bremek. (1963) 53, non Psychotria puberula C. Wright ex Griseb. (1866) 135. - Type: Perrier de la Bâthie 3757 (holo P; iso P), Madagascar, Domaine de l'Ouest, base ouest du massif de Manongarivo, près de Betofo.

Distribution - NW Madagascar.

36. Psychotria decumbens (Bremek.) A.P. Davis \& Govaerts, comb. nov.

Basionym: Mapouria decumbens Bremek. (1963) 54. - Type: Perrier de la Bâthie 16767 (holo P), Madagascar, Domaine du Centre, Tampoketsa entre Ikopa et Betsiboka, Manerinerina, $1500 \mathrm{~m}$.

Distribution - Central Madagascar.

37. Psychotria simianensis A.P. Davis \& Govaerts, nom. nov.

Mapouria humilis Bremek. (1963) 55, non Psychotria humilis Hiern (1877) 198. - Type: Perrier de la Bâthie 3965 (holo P; iso P), Madagascar, Domaine de l'Est, sur la rivière Simiana, 100 m.

Distribution - E Madagascar.

\section{SERIES VII}

38. Psychotria leilae A.P. Davis \& Govaerts, nom. nov.

Mapouria microcephala Bremek. (1963) 56, non Psychotria microcephala Miq. (1857) 293. - Type: Perrier de la Bâthie 3814 (holo P), Madagascar, Domaine de l'Ouest, massif de Manongarivo, $1600 \mathrm{~m}$.

Distribution - NW Madagascar.

Note - Psychotria leilae is named after the daughter of A. Davis.

39. Psychotria boenyana (Bremek.) A.P. Davis \& Govaerts, comb. nov.

Basionym: Mapouria boenyana Bremek. (1963) 57. - Type: Perrier de la Bâthie 3685 (holo P), Madagascar, Domaine de l'Ouest, Boeny, Ankarafantsika.

Distribution - W Madagascar.

40. Psychotria tsaratananensis (Bremek.) A.P. Davis \& Govaerts, comb. nov.

Basionym: Mapouria tsaratananensis Bremek. (1963) 58. - Type: Perrier de la Bâthie 3589 (holo P), Madagascar, Domaine du Centre (Sous-domaine Nord), Mt Tsaratanana, $1800 \mathrm{~m}$.

Distribution - N Madagascar.

40a. var. tsaratananensis

Distribution - N Madagascar. 
40b. var. pubescens (Bremek.) A.P. Davis \& Govaerts, comb. nov.

Basionym: Mapouria tsaratananensis Bremek. var. pubescens Bremek. (1963) 58. - Type: Humbert \& Capuron 25382 (holo P), Madagascar, Domaine du Centre (Sous-domaine Nord), montagnes au nord de Mangindrano (haute Maevarano) jusqu'au sommet d'Ambohimirahavavy, vallée d'Ambatohalo, 1200-1400 m.

Distribution - N Madagascar.

41. Psychotria manambolensis A.P. Davis \& Govaerts, nom. nov.

Mapouria humbertii Bremek. (1963) 59, non Psychotria humbertii Bremek. (1963) 157. - Type: Humbert 12105 (holo P; iso P), Madagascar, Domaine du Centre (Sud), entre le col du Kalambatitra et la vallée de la Manambolo, 1200-1400 m.

Distribution - Central and S Madagascar.

42. Psychotria ruhsamiana A.P. Davis \& Govaerts, nom. nov.

Mapouria tenuifolia Bremek. (1963) 60, non Psychotria tenuifolia Sw. (1788) 43. - Type: Perrier de la Bâthie 15480 (holo P), Madagascar (centre-nord), massif de Tsaratanana, $1500 \mathrm{~m}$.

Distribution - N Madagascar.

Note - This species is named after Markus Ruhsam, Verification Coordinator of the World Rubiaceae Checklist (www.kew.org/wcsp/rubiaceae).

43. Psychotria bathieana A.P. Davis \& Govaerts, nom. nov.

Mapouria tetramera Bremek. (1963) 61, non Psychotria tetramera Steyerm. (1972) 654. - Type: Perrier de la Bâthie 16282 (lectotype P, designated here), Madagascar, Domaine du Centre (Sousdomaine Nord), Mt Tsaratanana, $2000 \mathrm{~m}$.

Distribution - N Madagascar.

Note - This species is named after the French botanist J.H.M.A. Perrier de la Bâthie. Bremekamp (1963: 61) cites two specimens for the type of M.tetramera, Perrier de la Bâthie 16282 and 15374, the latter followed by the statement 'co-type fructifère'. We here designate Perrier de la Bâthie 16282 as the lectotype.

44. Psychotria imerinensis (Bremek.) A.P. Davis \& Govaerts, comb. nov.

Basionym: Mapouria imerinensis Bremek. (1963) 61. - Type: Perrier de la Bâthie 3561 (holo P), Madagascar, Domaine du Centre, Imerina Nord, Manankazo au nord-est d'Ankazobe, $1500 \mathrm{~m}$.

Distribution - Central Madagascar.

45. Psychotria mandrarensis (Bremek.) A.P. Davis \& Govaerts, comb. nov.

Basionym: Mapouria mandrarensis Bremek. (1963) 62. - Type: Humbert 6593 (holo P; iso P), Madagascar, Domaine du Centre, bassin supérieur du Mandrare, col et sommet de Marosoui, $1000-1400 \mathrm{~m}$.

Distribution - Central Madagascar. 
46. Psychotria distichodoma (Bremek.) A.P. Davis \& Govaerts, comb. nov.

Basionym: Mapouria distichodoma Bremek. (1963) 63. - Type: Perrier de la Bâthie 18366 (lectotype P, designated here), Madagascar, Domaine de l'Est, Ambatovola.

Distribution - E Madagascar.

Note - Bremekamp (1963: 64) cites two specimens for the type of M. distichodoma, Perrier de la Bâthie 18366 and 18367, the latter followed by the word 'co-type'. We here designate Perrier de la Bâthie 18366 as the lectotype.

47. Psychotria berizokae (Bremek.) A.P. Davis \& Govaerts, comb. nov.

Basionym: Mapouria berizokae Bremek. (1963) 64. - Type: Perrier de la Bâthie 421 (holo P), Madagascar, Domaine de l'Ouest, Berizoka.

Distribution - W Madagascar.

48. Psychotria ancaranensis (Bremek.) A.P. Davis \& Govaerts, comb. nov.

Basionym: Mapouria ancaranensis Bremek. (1963) 65. - Type: Humbert 19017 (holo P), Madagascar (Nord), prov. de Diégo-Suarez, collines et plateaux calcaires de l'Ankarana, forêt tropophile près d'Ambodimagodro.

Distribution - N Madagascar.

\section{Psychotria retiphlebia Baker}

Psychotria retiphlebia Baker (1887) 485. - Uragoga retiphlebia (Baker) Drake (1897) t. 428. - Mapouria retiphlebia (Baker) Bremek. (1963) 66. - Type: Baron 4908 (lectotype K, designated here; isolectotype P), Madagascar.

Distribution - Central Madagascar.

Note - Baker (1887: 485) cites two syntypes for P. retiphlebia, Baron 4285 and 4908. Bremekamp (1963: 67) also cites these two specimens, adding duplicates for Paris (P) and 'co-type' after the citation. We here designate Baron 4908 as the lectotype.

\section{Psychotria reducta Baker}

Psychotria reducta Baker (1887) 484. - Mapouria reducta (Baker) Bremek. (1963) 68. - Type: Baron 1029 (lectotype K, designated here; isolectotypes K, P), Central Madagascar.

Distribution - Central Madagascar.

Note - Baker (1887: 484-485) cites eight syntypes for P. reducta, Baron 1029, 1778, 1784, 3760, 3997, 4016, 4018, 4399. Bremekamp (1963: 68) cites only one specimen, Baron 1029, indicating a duplicate in the Paris herbarium ('Baron $1029 \mathrm{P}$, $\mathrm{K}$ [sic]). We here designate Baron 1029 (K) as the lectotype.

\section{0a. var. reducta}

Distribution - Central Madagascar. 
50b. var. pilosula (Bremek.) A.P. Davis \& Govaerts, comb. nov.

Basionym: Mapouria reducta (Baker) Bremek. var. pilosula Bremek. (1963) 69. - Type: Decary 13773 (holo P), Madagascar, Domaine du Centre, Ambositra.

Distribution - Central Madagascar.

51. Psychotria isalensis (Bremek.) A.P. Davis \& Govaerts, comb. nov.

Basionym: Mapouria isalensis Bremek. (1963) 69. - Type: Humbert 19571 (holo P; iso P), Madagascar, Domaine du Centre (Sud), plateaux et vallées de l'Isalo, à l'ouest de Ranohira, 800-1250 m.

Distribution - Central Madagascar.

52. Psychotria andetrensis (Bremek.) A.P. Davis \& Govaerts, comb. nov.

Basionym: Mapouria andetrensis Bremek. (1963) 71. - Type: Humbert 6850 (holo P), Madagascar, Domaine du Centre, haute vallée du Mandrare, prés d'Andetra, $800 \mathrm{~m}$.

Distribution - Central Madagascar.

\section{Psychotria parkeri Baker}

Psychotria parkeri Baker (1887) 485. - Mapouria parkeri (Baker) Bremek. (1963) 71. - Type: Parker s.n. (lectotype K, designated by Bremekamp (1963: 72), Madagascar, Domaine du Centre, Andrangaloaka.

Distribution - Central Madagascar.

Note - Baker (1887: 485) cites three syntypes for P. parkeri, Baron 1585, 3527, and Parker s.n. ('Also gathered in the forest of Andrangaloaka by Dr. Parker'; Baker (1887: 485)). Bremekamp (1963:72) cites only one type for Mapouria parkeri: 'Parker s.n. K'.

54. Psychotria distinctinervia A.P. Davis \& Govaerts, nom. nov.

Mapouria reticulata Bremek. (1963) 73, non Psychotria reticulata Ruiz \& Pav. (1799) 56. - Type: Perrier de la Bâthie 3588 (holo P), Madagascar, Domaine du Centre, Mt Tsaratanana, $2700 \mathrm{~m}$.

Distribution - N Madagascar.

55. Psychotria morley-smithiae A.P. Davis \& Govaerts, nom. nov.

Mapouria grandiflora Bremek. (1963) 74, non Psychotria grandiflora H. Mann (1867) 170. - Type: Humbert 18395 (holo P), Madagascar, Domaine du Centre, massif du Tsaratanana, Rés. Nat. IV, flanc sud de l'Antsianongatalata, $2600 \mathrm{~m}$.

Distribution - N Madagascar.

Note - This species is named after Anne Morley-Smith, who indefatigably provides logistic support for staff and visitors at the Royal Botanic Gardens, Kew. 
56. Psychotria ratovoarisonii A.P. Davis \& Govaerts, nom. nov.

Mapouria angustifolia Bremek. (1963) 75, non Psychotria angustifolia Poir. (1804) 703. - Type: Ratovoarison RN 1309 (holo P), Madagascar, limite des Domaines de l'Est et du Centre, prov. d'Andovoranto, distr. Moramanga, Périnet.

Distribution - E Madagascar.

Note - This species is named after Malagasy botanist F. Ratovoarison.

57. Psychotria anjanaharibensis A.P. Davis \& Govaerts, nom. nov.

Mapouria apoda Bremek. (1963) 76, non Psychotria apoda Steyerm. (1972) 668. - Type: Humbert, Capuron \& Cours 24607 (holo P), Madagascar, Domaine du Centre, massif de l'Anjanaharibe à l'ouest d'Andapa (haute Andramonta, bassin de la Lokoho), 1200-1400 m.

Distribution - NE Madagascar.

58. Psychotria manampanihensis (Bremek.) A.P. Davis \& Govaerts, comb. nov.

Basionym: Mapouria manampanihensis Bremek. (1963) 77. - Type: Humbert 6023 (holo P), Madagascar, Domaine du Centre, bassin de la Manampanihy, col de Fitana, 300-700 m.

Distribution - Central Madagascar.

59. Psychotria bemarivensis A.P. Davis \& Govaerts, nom. nov.

Mapouria calophylla Bremek. (1963) 78, non Psychotria calophylla Standl. (1916) 129. - Type: Perrier de la Bâthie 3596 (holo P), Madagascar, Domaine du Centre, Bemarivo, haute Androranga, $800 \mathrm{~m}$.

Distribution - Central Madagascar.

60. Psychotria viguieri (Bremek.) A.P. Davis \& Govaerts, comb. nov.

Basionym: Mapouria viguieri Bremek. (1963) 78. - Type: Perrier de la Bâthie 16283 (holo P), Madagascar, Domaine du Centre, massif du Tsaratanana, $2000 \mathrm{~m}$.

Distribution - N Madagascar.

61. Psychotria bridsoniae A.P. Davis \& Govaerts, nom. nov.

Mapouria magnifolia Bremek. (1963) 79, non Psychotria magnifolia Merr. (1921) 453. - Type: Perrier de la Bâthie 15282 (holo P), Madagascar, Domaine du Centre, massif du Tsaratanana, $1800 \mathrm{~m}$.

Distribution - N Madagascar.

Note - Named after English botanist and Rubiaceae specialist Diane M. Bridson, recently retired from the Royal Botanic Gardens, Kew.

62. Psychotria miae A.P. Davis \& Govaerts, nom. nov.

Mapouria assimilis Bremek. (1963) 80, non Psychotria assimilis Bremek. (1963) 154. - Type: Perrier de la Bâthie 3761 (holo P), Madagascar, Domaine du Centre, Andringitra, $1600 \mathrm{~m}$. 
Distribution - Central Madagascar. tion.

Note - This species is named after Mia Nachtergaele, the second author's inspira-

63. Psychotria retusa (Bremek.) A.P. Davis \& Govaerts, comb. nov.

Basionym: Mapouria retusa Bremek. (1963) 81. - Type: Perrier de la Bâthie 3733 (holo P; iso P), Madagascar, Domaine du Sambirano, haut Sambirano, $800 \mathrm{~m}$.

Distribution - NW Madagascar.

64. Psychotria rakotoniaina A.P. Davis \& Govaerts, nom. nov.

Mapouria laxiflora Bremek. (1963) 82, non Psychotria laxiflora Blume (1826) 964. - Type: Rakotoniaina RN 5355 (holo P), Madagascar, Domaine de l'Est, distr. Tamatave, Ambodiriana, Rés. Nat. I.

Distribution - E Madagascar.

Note - This species is named after Malagasy botanist and plant collector F. Rakotoniaina.

65. Psychotria suarezensis A.P. Davis \& Govaerts, nom. nov.

Mapouria acuminata Bremek. (1963) 82, non Psychotria acuminata Benth. (1845) 107. - Type: Humbert 19076 (holo P; iso P), Madagascar (Nord), prov. de Diégo-Suarez, collines et plateaux calcaires de l'Ankarana, Analamahitso, $350 \mathrm{~m}$.

Distribution - N Madagascar.

66. Psychotria calothyris (Bremek.) A.P. Davis \& Govaerts, comb. nov.

Basionym: Mapouria calothyris Bremek. (1963) 83. - Type: Humblot 1462 (holo P), Comores, Anjouan.

Distribution - Comoros (Anjouan).

67. Psychotria antsalovensis (Bremek.) A.P. Davis \& Govaerts, comb. nov.

Basionym: Mapouria antsalovensis Bremek. (1963) 84. - Type: Léandri, Capuron \& Razafindrakoto 2123 (holo P; iso P), Madagascar, Domaine de l'Ouest, calcaires de l'Antsingy, vers Ambodiriana (est d'Antsalova), $100-150 \mathrm{~m}$.

Distribution - W Madagascar.

Note - Bremekamp (1963: 15,84) expressed some doubts as to whether this species should be placed in Mapouria, and it was cited by him as 'Mapouria? antsalovensis' . His circumspection was mainly due to the lack of fruit and seed; the only collections known to him were of flowering material (Léandri, Capuron \& Razafindrakoto 2123 (P) and Razafindrakoto RN $11684(\mathrm{P}))$. Mapouria antsalovensis has been recollected recently (e.g. Jongkind et al. 3265 (K, WAG), Labat \& Deroin 2285 (K, P)) but it is still only known from flowering material. Based on the material at hand, and having a good knowledge of other Madagascan Rubiaceae genera, we believe that $M$. antsalovensis belongs to Psychotria and the necessary new combination is made. 


\section{NOTES}

\section{Psychotria mesentericarpa Baker}

Psychotria mesentericarpa Baker (1885) 412. - Type: Baron 2969 (lectotype K, designated here), Central Madagascar.

Distribution - Central Madagascar.

Note - Baker (1885: 412) cites five syntypes for P. mesentericarpa, including Baron $851,1240,2969,2995,3015$. According to Bremekamp (1963) P. mesentericarpa is a 'nomen confusum'; he considered Baron 1240 to be M. reducta and Baron 851 and 2995 to be M. parkeri (Bremekamp, 1963: 68, 71-73; and as labelled on specimens). Bremekamp (1963: 68) did not place Baron 2969 and 3015 with either of the aforementioned species: on herbarium specimens he determined them as M. boenyana cf. [sic]. These specimens differ from $P$. reducta and $P$. parkeri in the presence of short hairs on the undersurface of the leaf (vs glabrous) and a more or less repand calyx rim (vs lobed), although there may be other differences. Further work may show that $P$. mesentericarpa is conspecific with $P$. parkeri, but for the time being we recognize them as distinct. We take the opportunity here of lectotypifying P. mesentericarpa with the specimen Baron 2969.

\section{ACKNOWLEDGEMENTS}

We would like to thank the Muséum National d'Histoire Naturelle, Paris (P), for placing herbarium material of the study group at our disposal. We are also grateful to the Director and staff at Paris, and particularly Jean-Noël Labat, for providing research facilities during extended study visits. This work would not have been possible without the cooperation of the following organisations and ministries in Antananarivo, Madagascar: Association National pour la Gestion des Aires Protégées (ANGAP), Ministère des Eaux et Forêts (MEF), Ministère de la Recherche Scientifique Centre National de Recherche Appliquée au Développement Rural (FOFIFA). At Kew, we would like to thank Diane Bridson for her suggestions and advice regarding Psychotria and Psychotrieae. At the National Botanic Garden of Belgium, Meise, we would like to thank Elmar Robbrecht for a careful review of an earlier version of this paper. We are very grateful to the Leverhulme Trust and the Global Biodiversity Information Facility (GBIF), within the Electronic Catalogue of Names of Known Organisms (ECAT) programme, for providing funding for our study.

\section{REFERENCES}

Andersson, L. 2002. Relationships and generic circumscriptions in the Psychotria complex (Rubiaceae, Psychotrieae). Syst. Geogr. Pl. 72: 167-202.

Aublet, J.B.C. 1775a. Histoire des Plantes de la Guiane François, vol. 1: 175. Didot Jeune, Paris. Aublet, J.B.C. 1775b. Histoire des Plantes de la Guiane François, vol. 3: 1-193. Didot Jeune, Paris. Baker, J.G. 1885. Further contributions to the flora of Madagascar. Second and final part. J. Linn. Soc., Bot. 21: 407-455.

Baker, J.G. 1887. Further contributions to the flora of Madagascar. J. Linn. Soc., Bot. 22: 441537.

Bentham, G. 1845. The botany of the voyage of H.M.S Sulphur, part 5: 107. Smith Elder \& Co., London.

Blume, C.L. 1823. Catalogus: 54. Batavia (Jakarta).

Blume, C.L. 1826. Bijdragen tot de Flora van Nederlandsch Indië: 943-1018. Lands Drukkerij, Batavia (Jakarta). 
Bremekamp, C.E. B. 1960. Les «Psychotria» bactériophiles de Madagascar. Notul. Syst. (Paris) 16: 41-54.

Bremekamp, C.E.B. 1961. The identity of the genera Mapouria Aubl. and Grumilea Gaertn. (Rubiaceae, Psychotrieae). Acta Bot. Neerl. 10: 307-319.

Bremekamp, C.E.B. 1963. Sur quelques genres de Psychotriées (Rubiaceés) et sur leurs représentants Malgaches et Comoriens. Verh. Kon. Ned. Akad. Wetensch., Afd. Natuurk., Tweede Sect. 54, 5: $1-181$.

Davis, A.P., D.M. Bridson, C. Jarvis \& R. Govaerts. 2001. The typification and characterization of the genus Psychotria L. (Rubiaceae). Bot. J. Linn. Soc. 135: 35-42.

Drake del Castillo, M.E. 1897. Histoire physique naturelle et politique de Madagascar - Histoire naturelle des plantes, vol. 36, 6, Atlas 4. Renauld \& Cadot, Paris.

Elmer, A.D.E. 1913. Rubiaceae from Mount Urdaneta. Leafl. Philipp. Bot. 5: 1855-1905.

Grisebach, A.H.R. 1866. Catalogus Plantarum Cubensium: 121-143. Engelmann, Leipzig.

Hamilton, C.W. 1989. A revision of Mesoamerican Psychotria subgenus Psychotria (Rubiaceae), part 1: Introduction and species 1-16. Ann. Missouri Bot. Gard. 76: 67-111.

Hiern, W.P. 1877. In: D. Oliver (ed.), Flora of Tropical Africa vol. 3: 33-247. Reeve \& Co., Ashford.

Holmgren, P.K., N.H. Holmgren \& L.C. Barnett. 1990. Index Herbariorum. In: Part 1: The Herbaria of the World, 8th Edition. Regnum Vegetabile [series]. New York Botanical Garden, New York.

Kunth, C.S. 1819. In: A. de Humboldt, A. Bonpland \& C.S. Kunth, Nova Genera et Species Plantarum, Vol. 3: 358. Sumtibus Librairie Graeco-Latini-Germanicae, Paris.

Mann, H. 1867. Rubiaceae. Enumeration of Hawaiian plants. Proc. Amer. Acad. Arts 7: 169-172. Welch, Bigelow \& Co., Cambridge.

Merrill, E.D. 1921 ('1920’). Studies on Philippine Rubiaceae, IV. Philipp. J. Sci. 17: 425-485.

Miquel, F. A.W. 1857. Flora van Nederlandsch Indië, vol. 2: 129-356. Fleischer, Leipzig.

Müller Argoviensis, J. 1881. In: C.F.P. von Martius (ed.), Flora Brasiliensis vol. 6, 5: 237, 249. Fleischer, Leipzig.

Petit, E. 1964. Les espèces Africaines du genre Psychotria L. (Rubiaceae) - I. Bull. Jard. Bot. Belg. 34: 1-229.

Piesschaert, F. 2001. Carpology and pollen morphology of the Psychotrieae (Rubiaceae-Rubioideae). $\mathrm{PhD}$ thesis, Katholieke Universiteit Leuven, Belgium.

Poiret, J.L.M. 1804. In: J.B.A.P.M de Lamarck, Encyclopédie Méthodique. Botanique vol. 5: 703. Agasse, Paris.

Robbrecht, E. 1988. Tropical woody Rubiaceae. Characteristic features and progressions. Contributions to a new subfamilial classification. Opera Bot. Belg. 1: 1-271.

Robbrecht, E. 1989. A remarkable new Chazaliella (African Psychotrieae), exemplifying the taxonomic value of pyrene characters in the Rubiaceae. Bull. Mus. Natl. Hist. Nat., sér. 4, 11, B, Adansonia 4: 341-349.

Rolfe, R.A. 1893. Enumeration of the flowering plants and ferns of St. Vincent and adjacent islets. Bull. Misc. Inform. Kew 81: 235-296.

Ruiz, H. \& J. A. Pavon. 1799. Flora Peruvianae et Chilensis Prodromus vol. 2: 56, 59. Typis Gabriels de Sancha, Madrid.

Schumann, K. M. 1898. In: F.V. Reinecke, Die Flora der Samoa-Inseln. Teil II: Siphonogamen. Bot. Jahrb. Syst. 25: 578-708.

Schumann, K.M. 1899. Rubiaceae africanae. Bot. Jahrb. Syst. 28: 55-113.

Standley, P.C. 1916. New species of Psychotria from Panama. Contr. U.S. Natl. Herb. 18: 128-133.

Steyermark, J. A. 1972. Rubiaceae. In: B. Maguire et al. (eds.), The botany of the Guayana Highland - Part IX. Mem. New York Bot. Gard. 23: 227-832.

Swartz, O.P. 1788. Nova Genera \& Species Plantarum seu Prodromus: 40-55. Holmiae, Upsaliae $\&$ Aboae.

Taylor, C.M. 1996. Overview of the Psychotrieae (Rubiaceae) in the Neotropics. Opera Bot. Belg. 7: 261-270.

Urban, I. 1913. Symbolae Antillanae 7: 433-477. Borntraeger, Leipzig. 


\section{INDEX}

The numbers behind the names refer to the species number in this article. Accepted names are in roman type; new combinations and new names in bold; synonyms and basionyms in italics. The authors' names A.P. Davis \& Govaerts are abbreviated here as D \& G.

\section{Mapouria}

acuminata Bremek. 65

aegialodes Bremek. 30

ambongensis Bremek. 17

ancaranensis Bremek. 48

andetrensis Bremek. 52

angustifolia Bremek. 56

ankafinensis (K. Schum.) Bremek. 15

antsalovensis Bremek. 67

apoda Bremek. 57

assimilis Bremek. 62

baronii Bremek. 16

berizokae Bremek. 47

betamponensis Bremek. 23

boenyana Bremek. 39

calophylla Bremek. 59

calothyris Bremek. 66

capitata Bremek. 18

decumbens Bremek. 36

discolor Bremek. 1

distichodoma Bremek. 46

dolichocarpa Bremek. 26

erythrotricha Bremek. 33

glaucescens Bremek. 8

grandiflora Bremek. 55

himanthophylla Bremek. 9

humbertii Bremek. 41

humblotii Bremek. 19

humilis Bremek. 37

imerinensis Bremek. 44

isalensis Bremek. 51

lavanchiei Bremek. 27

laxiflora Bremek. 64

lokohensis Bremek. 2

longipedunculata Bremek. 13

macrochlamys Bremek. 32

magnifolia Bremek. 61

manampanihensis Bremek. 58

mandrarensis Bremek. 45

mangorensis Bremek. 22

maroensis Bremek. 11

marojejensis Bremek. 14

megalocarpa Bremek. 21

menalohensis Bremek. 25

microcephala Bremek. 38

montana Bremek. 3

onivensis Bremek. 24

oreotrephes Bremek. 7

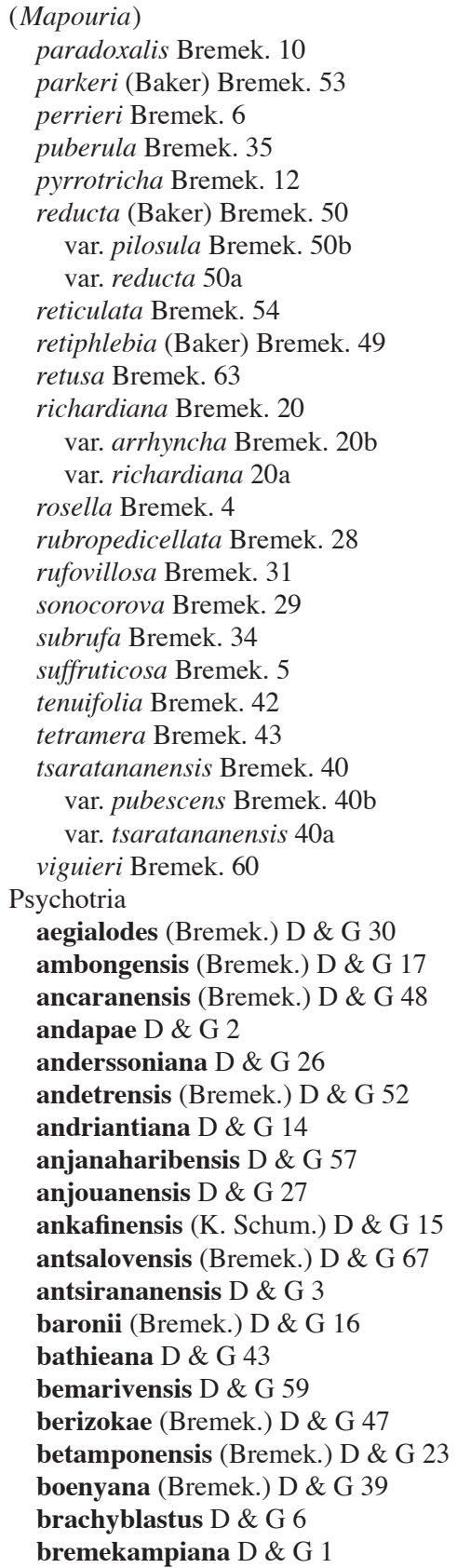

(Mapouria)

parkeri (Baker) Bremek. 53

perrieri Bremek. 6

reducta (Baker) Bremek. 50

var. pilosula Bremek. 50b

var. reducta 50a

reticulata Bremek. 54

retiphlebia (Baker) Bremek. 49

retusa Bremek. 63

chardiana Bremek. 20

var. arrhyncha Bremek. 20b

rosella Bremek. 4

rubropedicellata Bremek. 28

rufovillosa Bremek. 31

sonocorova Bremek. 29

subrufa Bremek. 34

suffruticosa Bremek. 5

tenuifolia Bremek. 42

tetramera Bremek. 43

var. pubscens Bremek. 40b

viguieri Bremek. 60

sychotria

aegialodes (Bremek.) D \& G 30

ambongensis (Bremek.) D \& G 17

ancaranensis (Bremek.) D \& G 48

andetrensis (Bremek.) D \& G 52

andriantiana $D$ \& G 14

anjanaharibensis D \& G 57

anjouanensis D \& G 27

ankafinensis (K. Schum.) D \& G 15

antsalovensis (Bremek.) D \& G 67

antsirananensis $\mathrm{D} \& \mathrm{G} 3$

baronii (Bremek.) D \& G 16

berizokae (Bremek.) D \& G 47

betamponensis (Bremek.) D \& G 23

brachyblastus D \& G 6

bremekampiana D \& G 1 


\author{
(Psychotria) \\ bridsoniae D \& G 61 \\ calothyris (Bremek.) D \& G 66 \\ capuronii D \& G 5 \\ cephaloides D \& G 18 \\ decumbens (Bremek.) D \& G 36 \\ distichodoma (Bremek.) D \& G 46 \\ distinctinervia D \& G 54 \\ glaucifolia $D$ \& $G 8$ \\ humblotii (Bremek.) D \& G 19 \\ imerinensis (Bremek.) D \& G 44 \\ integristipulata D \& G 34 \\ isalensis (Bremek.) D \& G 51 \\ leilae D \& G 38 \\ macrochlamys (Bremek.) D \& G 32 \\ manambolensis D \& G 41 \\ manampanihensis (Bremek.) D \& G 58 \\ mandrarensis (Bremek.) D \& G 45 \\ mangorensis (Bremek.) D \& G 22 \\ maningoryensis $D \& G$ \\ manongarivensis $D$ \& $\mathrm{G} 35$ \\ maroensis (Bremek.) D \& G 11 \\ megalocarpa (Bremek.) D \& G 21 \\ menalohensis (Bremek.) D \& G 25 \\ mesentericarpa Baker 68 \\ miae D \& G 62 \\ morley-smithiae D \& G 55 \\ nossibensis D \& G 20 \\ var. arrhyncha (Bremek.) D \& G 20b \\ var. nossibensis $20 \mathrm{a}$
}

(Psychotria)

onivensis (Bremek.) D \& G 24

oreotrephes (Bremek.) D \& G 7

paradoxalis (Bremek.) D \& G 10

parkeri Baker 53

pyrrotricha (Bremek.) D \& G 12

rakotonasoloi $D$ \& $G 13$

rakotoniaina $\mathrm{D} \& \mathrm{G} 64$

ratovoarisonii $D$ \& $G 56$

reducta Baker 50

var. pilosula (Bremek.) D \& G 50b

var. reducta $50 \mathrm{a}$

retiphlebia Baker 49

retusa (Bremek.) D \& G 63

rosella (Bremek.) D \& G 4

rubropedicellata (Bremek.) D \& G 28

rufovillosa (Bremek.) D \& G 31

ruhsamiana $D$ \& $G 42$

simianensis $D$ \& $G 37$

sonocorova (Bremek.) D \& G 29

suarezensis D \& G 65

tolongoinensis $D \&$ G 33

tsaratananensis (Bremek.) D \& G 40

var. pubescens (Bremek.) D \& G 40b

var. tsaratananensis $40 \mathrm{a}$

viguieri (Bremek.) D \& G 60

Uragoga

ankafinensis K. Schum. 15

retiphlebia (Baker) Drake 49 